\section{A transição de saúde pública 'internacional' para 'global' e a Organização Mundial da Saúde ${ }^{1}$}

\section{The transition from 'international' to 'global' public health and the World Health Organization}

Theodore M. Brown

Departamento de História Universidade de Rochester, Nova York 368 Rush Rhees Library

Rochester, New York 14627-0070

Theodore_Brown@urmc.rochester.edu

\section{Marcos Cueto}

Facultat de Salud Publica y Administración Universidade Peruana Cayetano Heredia, Lima Malecon Paul Harris 260, depto 502 Barranco Lima - Peru mcueto@upch.edu.pe

Elizabeth Fee

Divisão de História da Medicina da National Library of Medicine, Bethesda Buildin 38, Room 1E21 8600 Rockville Pike Bethesda 20894 MD, USA fee@mail.nih.gov
BROWN, Th. M.; CUETO, M.; FEE, E.: A transição de saúde pública 'internacional' para 'global' e a Organização Mundial da Saúde. História, Ciências, Saúde - Manguinhos, v. 13, n. 3, p. 623-47, jul.-set. 2006.

No contexto da saúde pública internacional, 'saúde global' parece estar emergindo como um termo de reconhecida preferência. Este artigo apresenta uma análise crítica do significado e importância de 'saúde global', e situa sua crescente popularidade no contexto histórico. Um foco específico deste estudo é o papel da Organização Mundial da Saúde OMS, tanto na saúde 'internacional' quanto na 'global', e como um agente na transição de uma para outra. Entre 1948 e 1998 a OMS enfrentou dificuldades, ao deparar com uma crise organizacional, cortes orçamentários e status diminuído, especialmente em face da crescente influência de novos e poderosos atores, como o Banco Mundial. Sugerimos que a OMS começou a remodelar-se e a reposicionar-se nos papéis de coordenação, planejamento estratégico e liderança de iniciativas de 'saúde global', em resposta a esse contexto internacional em transformação.

PALAVRAS-CHAVE: saúde pública internacional; saúde pública global; Organização Mundial da Saúde.

BROWN, Th. M.; CUETO, M.; FEE, E.: The transition from 'international' to 'global' public health and the World Health Organization. História, Ciências, Saúde - Manguinhos, v. 13, n. 3, p. 623-47, July-Sept. 2006.

Within the context of international public health, 'global health' seems to be emerging as a recognized term of preference. This article presents a critical analysis of the meaning and importance of 'global health' and situates its growing popularity within a historical context. A specific focus of this work is the role of the World Health Organization - WHO in both 'international' and 'global' health, and as an agent of transition from one to the other. Between 1948 and 1998, the WHO went through a period of hardship as it came up against an organizational crisis, budget cuts and a diminished status, especially when confronted with the growing influence of new, powerful players like the World Bank. We suggest that the WHO has responded to this changing international context by initiating its own process of restructuring and repositioning as an agent for coordinating, strategically planning and leading 'global health' initiatives.

KEYWORDS: international public health; global public health; World Health Organization. 
* Tradução de Annabella Blyth. Revisão técnica de Gilberto Hochman

\begin{abstract}
A té mesmo um rápido olhar pelos títulos de livros e artigos na literatura recente sobre medicina e saúde pública sugere que uma importante transição encontra-se em curso. Os termos 'global', 'globalização' e suas variantes estão em toda parte, e, no contexto específico da saúde pública, 'global' parece estar emergindo como um termo de reconhecida preferência. ${ }^{2}$ Como um indicador, o número de entradas sob as rubricas saúde 'global' e 'internacional' nas bases de dados da literatura especializada (como PubMed) mostra que saúde 'global' encontra-se em rápido crescimento, aparentemente tendo ultrapassado saúde 'internacional' em algum momento de 2005 (Tabela 1). Apesar de universidades, agências governamentais e entidades filantrópicas privadas dos países desenvolvidos estarem todas usando o termo de modo muito visível, a origem e o significado da expressão 'saúde global' ainda não estão claros. ${ }^{3}$
\end{abstract}

Tabela I - Número total de entradas no PubMed sob as rubricas International e Global, por década

[Busca por palavras-chave, outubro de 2005]

\begin{tabular}{ccc}
\hline Década & International Health* & Global Health* \\
\hline 1950 & 1.007 & 54 \\
1960 & 3.303 & 155 \\
1970 & 8.369 & 1.137 \\
1980 & 16.924 & 7.176 \\
1990 & 49.158 & 27.794 \\
$2000-$ set./2005 & $54.540 * *$ & $41.623 * *$ \\
\hline
\end{tabular}

* Esta contagem considera terminações variantes, por exemplo, internationalize e internationalization; globalize e globalization.

** Note-se que a contagem abrange apenas 55 meses.

O propósito deste artigo é oferecer uma visão panorâmica e histórica sobre o surgimento da terminologia 'saúde global'. Acreditamos que um estudo dessa mudança lingüística trará frutos importantes, não apenas sobre modismos no uso da linguagem. Nossa tarefa aqui é fazer uma análise crítica do significado, da emergência e da importância do termo 'saúde global' e situar sua crescente popularidade em um contexto histórico mais amplo. Um foco específico deste artigo é o papel da Organização Mundial da Saúde - OMS tanto na saúde 'internacional' quanto na 'global' e como um agente na transição de uma para outra.

Inicialmente, vamos definir e diferenciar alguns termos essenciais. Saúde 'internacional' era um termo usado com considerável freqüência já no final do século XIX e no início do século XX, e referia-se especialmente a um foco no controle de epidemias ultrapassando fronteiras entre nações, ou seja, 'inter-nacionalmente'. 'Intergovernamental' se refere às relações entre governos de nações 
soberanas, neste caso com relação às políticas e práticas de saúde pública. Saúde 'global', em geral, indica a consideração das necessidades de saúde da população de todo o planeta, acima dos interesses de nações em particular. $O$ termo 'global' também é associado à crescente importância de atores para além de agências e organizações governamentais e intergovernamentais - por exemplo, a mídia, fundações influentes internacionalmente, corporações transnacionais. Logicamente, os termos 'internacional', 'intergovernamental' e 'global' não são mutuamente excludentes e, de fato, podem ser entendidos como complementares. Assim, poderíamos dizer que a OMS é uma agência intergovernamental que desempenha funções internacionais com o objetivo de melhorar a saúde global.

Feitas essas definições, não deve surpreender o fato de que saúde 'global' não seja uma invenção inteiramente de anos recentes. 'Global' era usado, por vezes, bem antes dos anos 90, como no "programa de erradicação global da malária" conduzido pela OMS em meados dos anos 50; em um folheto de 1958 do Public Affairs Committee (Comitê de Assuntos Públicos) da OMS, The World Health Organization: its global battle against disease" (A Organização Mundial da Saúde: sua luta global contra doenças; Deutsch, 1958); num relatório de 1971 sobre The politics of global health (A política de saúde global) para o Congresso dos Estados Unidos (U. S. House of Representatives, 1971; Packard, 1998) e em muitos estudos sobre o "problema da população global" na década de 1970 (por exemplo, Wilson, 1974). Mas seu uso era geralmente limitado, e em declarações e documentos oficiais era apenas esporádico, quase sempre ligado ao temor das pandemias. Hoje cresce o número de referências à saúde 'global' (Banta, 2001). Ainda assim, as perguntas permanecem: quantos participaram nessa mudança de terminologia, e se estes a consideram modismo, algo trivial ou diferencial.

Essas perguntas atraíram a atenção de Supinda Bunyavanich e Ruth B. Walkup, que publicaram, com o título provocativo de $U$. S. public health leaders shift toward a new paradigm of global health (Líderes da saúde pública norte-americana mudam em direção a um novo paradigma de saúde global), seu relatório de entrevistas realizadas em 1999 com 29 "líderes de saúde internacional" (Bunyavanich \& Walkup, 2001). Seus depoentes dividiram-se em dois grupos. Cerca de metade considerou que não havia necessidade de uma nova terminologia e que o rótulo 'saúde global' era jargão sem significado. A outra metade pensava haver profundas diferenças entre saúde 'internacional' e 'global', e que 'global' significava claramente algo transnacional. Apesar de esses últimos sentirem ter ocorrido uma mudança importante no decorrer dos últimos anos, eles pareciam incapazes de articular ou definir claramente essa mudança. 
Em 1998, Derek Yacht e Douglas Bettcher aproximaram-se da compreensão tanto da essência quanto da origem da nova 'saúde global' em um artigo de duas partes sobre The globalization of public health (A globalização da saúde pública), no American journal of public health (Yacht \& Bettcher, 1998a, b). Eles definiram o "novo paradigma" da globalização como "o processo de crescente interdependência e integração econômica, política e social, à medida que capital, bens, pessoas, conceitos, imagens, idéias e valores cruzam fronteiras nacionais". As raízes da globalização eram longas, diziam eles, remontando pelo menos ao século XIX, mas o processo estava assumindo uma nova magnitude no final do século XX. A globalização da saúde pública, argumentavam, tinha dois aspectos, um promissor e outro ameaçador.

No lado positivo, havia a difusão mais fácil de tecnologias úteis, comunicações rápidas e idéias e valores como direitos humanos. No lado negativo, havia riscos tais como a diminuição das redes sociais de segurança, a comercialização facilitada de tabaco, álcool e drogas psicoativas, a disseminação mundial facilitada de doenças infecciosas, e a rápida degradação ambiental com conseqüências perigosas para a saúde pública. Mas Yacht e Bettcher estavam convencidos de que a OMS poderia transformar esses riscos em oportunidades. Essa organização poderia ajudar a criar informação e sistemas de vigilância mais eficientes ao reforçar seu sistema global de monitoramento e alerta, criando assim "sistemas globais de alerta antecipado". Até as nações mais poderosas comprariam a idéia desse novo sistema mundial globalmente interdependente, quando percebessem que tal envolvimento seria de seu interesse.

Apesar da longa lista de problemas e ameaças, Yacht e Bettcher foram bastante acríticos ao promoverem as virtudes da saúde pública global e o papel de liderança da OMS. Num editorial no mesmo número da revista, George Silver observou que Yacht e Bettcher trabalhavam para a OMS e que sua posição era similar a outras posições otimistas adotadas por funcionários e defensores da OMS. Mas Silver ressaltou que a OMS estava, de fato, num mau momento: "o papel de liderança da OMS passou para o Banco Mundial, muito mais rico e inflwente, e a missão da OMS se dispersou por outras agências da Organização das Nações Unidas - ONU" (Silver, 1998, p.728). Países doadores ricos estavam devendo bilhões de dólares, o que deixava a ONU e suas agências em "desordem, paralisadas por restrições financeiras e incompetências internas, frustradas por guerras de conhecimento e políticas nacionais". Dada essa realidade, a posição de Yacht e Bettcher de promover a 'saúde pública global', enquanto associados à OMS, era, no mínimo, intrigante. Por que esses porta-vozes da tão criticada OMS, que aparentemente se encontrava em situação tão difícil, estavam tão otimistas com relação à saúde pública 'global'? 


\section{A OMS, primeiros anos}

Para compreender melhor o papel de Yacht e Bettcher e, de modo mais geral, o da OMS, será útil revisar a história da organização de 1948 a 1998, na medida em que nessa época deixou seu papel de líder inquestionável da saúde internacional para buscar seu lugar no disputado mundo da 'saúde global'.

A OMS teve início formalmente em 1948, quando a primeira Assembléia Mundial da Saúde, realizada em Genebra, ratificou sua criação. A idéia de uma instituição permanente para a saúde internacional remonta a 1902, quando da organização do International Sanitary Office of the American Republics (Repartição Sanitária Internacional das Repúblicas Americanas), denominada a partir de 1920 como Repartição de Saúde Panamericana, com base em Washington (DC), a qual algumas décadas depois tornou-se a Repartição Sanitária Pan-Americana e, finalmente, a partir de 1959, Organização PanAmericana da Saúde (OPS, 1992; Cueto, 2004a). A Fundação Rockefeller, especialmente sua International Health Division (Divisão Internacional de Saúde), criada em meados da década de 1910, também foi um importante ator na saúde internacional no início do século XX (ver Farley, 2003; Birn, 1996; Cueto, 1994).

Duas agências internacionais de saúde baseadas na Europa também foram importantes. Uma delas foi o Office Internationale d'Hygiène Publique (Escritório Internacional de Higiene Pública), que funcionou em Paris a partir de 1907, concentrando-se em diversas atividades básicas relacionadas à administração de acordos sanitários internacionais e à rápida troca de informações epidemiológicas (Office International D'Hygiène Publique; 1933; Basch, 1991; Aykroyd, 1968). A segunda agência, a League of Nations Health Organization (Organização da Saúde da Liga das Nações), iniciou seus trabalhos em 1920 (Bourdreau, 1929; 1944; Howard-Jones, 1978; Dubin, 1995). Essa organização estabeleceu sua sede em Genebra, na Suíça, patrocinou uma série de comissões internacionais sobre doenças e publicou estudos epidemiológicos e relatórios técnicos. Entre 1921 e 1939 o diretor médico da seção de higiene da organização da Liga, o polonês Ludwik Rajchman, deu ênfase à medicina social, assinalando que as patologias tinham origem nas condições de vida como a pobreza, habitação inadequada e má nutrição (Balinska, 1998). A Organização da Saúde da Liga das Nações tinha orçamento pequeno e defrontou-se com oposição velada de outras organizações nacionais e internacionais, inclusive do Serviço de Saúde Pública dos Estados Unidos. Apesar dessas complicações, que limitavam a eficácia da Liga, tanto o Office Internationale d'Hygiène Publique quanto a Health Organization sobreviveram durante a Segunda Guerra Mundial, e estiveram presentes no crítico momento do pós-guerra, quando seria definido o futuro da saúde 
internacional. Mesmo assim a Repartição Sanitária Panamericana se renovou no final da década de 1940 com a eleição de Fred L. Soper, ex-funcionário da Fundação Rockefeller que havia trabalhado muitos anos no Brasil, para o cargo de Diretor. Por seu lado a International Health Division da Fundação Rockefeller estava por se retirar de uma ativa participação na saúde internacional. Essa Divisão deixou de existir em 1951 (ver Farley, 2003).

Uma conferência internacional realizada em São Francisco em 1945, patrocinada pelos vencedores da Segunda Guerra Mundial, aprovou a criação das Nações Unidas e votou também pela imediata reunião para a criação de uma nova agência de saúde especializada (proposta do brasileiro Geraldo de Paula Souza e de Szeming Sze, delegado da China). Participantes da reunião inicialmente formaram uma comissão de indivíduos proeminentes, entre os quais se encontravam René Sand, da Bélgica, Andrija Stampar, da Iugoslávia, e Thomas Parran, dos Estados Unidos. Sand e Stampar eram amplamente reconhecidos no campo da medicina social. A comissão reuniu-se entre 1946 e início de 1948, para planejar a nova organização internacional de saúde. Representantes da Repartição Sanitária Pan-Americana, cujos líderes resistiram à encampação pela nova agência, também se envolveram, assim como os líderes de novas instituições; é o caso da United Nations Relief and Rehabilitation Administration - UNRRA (Administração das Nações Unidas para a Libertação e Reabilitação), que havia desenvolvido importantes campanhas de saúde na Europa e na Ásia nos anos finais da Segunda Guerra Mundial e na qual haviam trabalhado tanto Paula Souza como Zee.

Nesse contexto, a primeira Assembléia Mundial da Saúde ocorreu em Genebra, em junho de 1948, e criou formalmente a Organização Mundial da Saúde, como una agência especializada das Nações Unidas. O escritório parisiense, a Organização de Saúde da Liga das Nações e a UNRRA fundiram-se na nova agência. A Repartição Sanitária Pan-Americana, dirigida por Fred L. Soper, foi autorizada a manter sua condição de autonomia como parte de um esquema de regionalização (Parran, 1958; Soper, 1977; Siddiqi, 1995). A OMS dividiu formalmente o mundo em seis regiões para promover a descentralização: Américas, Sudeste Asiático, Europa, Mediterrâneo Oriental, Pacífico Ocidental e África, mas só veio a implementar essa regionalização na década de 1950. Embora uma visão 'internacional' e 'intergovernamental' tenha prevalecido nas décadas de 1940 e 1950, a denominação de Organização Mundial da Saúde também indicou uma perspectiva 'global'.

O primeiro Diretor Geral da OMS, Brock Chisholm, era um psiquiatra canadense identificado com a tradição da medicina social britânica que ele considerava diferente da medicina 'socializada' soviética. Os Estados Unidos, importante contribuinte no orçamento 
da OMS, desempenhou inicialmente certo papel contraditório: por um lado, apoiou o sistema das Nações Unidas em suas metas amplamente mundiais, mas, por outro lado, invejava sua soberania e manteve o direito de intervir unilateralmente nas Américas em nome da segurança nacional. Sua política se explica em parte pelo contexto político - início da Guerra Fria, quando as relações entre os Estados Unidos e a União Soviética começavam a se tornar tensas. Outro problema para a OMS foi que sua constituição tinha de ser ratificada por Estados nacionais, um lento processo que significou ter de buscar sua legitimidade através da oferta de sua colaboração na reconstrução dos sistemas de saúde dos países europeus destruídos pela guerra e por sua intervenção em uma série de problemas de saúde de caráter mundial (Seventh Meeting..., PASO, 1949). Como agência intergovernamental, a OMS tinha de responder ao contexto político mais amplo. A política da Guerra Fria apresentava uma relevância específica, com um inquestionável impacto na OMS em termos de políticas e de pessoal. Assim, quando, em 1949, a União Soviética e outros países comunistas se retiraram do sistema da ONU e, portanto, da OMS, os Estados Unidos e seus aliados puderam facilmente exercer uma influência dominante. Em 1953, Chilshom completou seu período como diretor geral e foi substituído pelo brasileiro Marcolino Candau. Candau havia trabalhado sob a direção de Soper (a quem chamava de 'comandante') no controle da malária no Brasil, e estava vinculado aos programas 'verticais' de controle de doenças da Fundação Rockefeller; posteriormente, apoiou também a adoção desses programas pela Repartição Sanitária Pan-Americana nos quais a transferência de tecnologia era um dos componentes principais das intervenções de saúde (WHO, s.d.; 1983). Candau foi diretor geral da OMS durante mais de vinte anos. No período entre 1949 e 1956, durante o qual a União Soviética retornou à $\mathrm{ONU}$ e à $\mathrm{OMS}$, esta última era percebida como estreitamente vinculada aos interesses dos Estados Unidos. O regresso da União Soviética coincidiu com o processo de desstalinização nesse país (Joseph Stálin morreu em 1953) e com a emergência, depois de três anos de lutas internas, de Nikita Khrushchev, o qual prometeu competir e ganhar os países capitalistas em seus próprios terrenos, o que incluía não apenas o armamento nuclear mas também a retórica pacifista e a capacidade científica e tecnológica (Zubok \& Pleshakov, 1996).

Em 1955, Candau foi encarregado de impulsionar a campanha da OMS para erradicação da malária, aprovada naquele ano pela Assembléia Mundial da Saúde realizada na Cidade do México. (Um ano antes a Assembléia da Repartição Sanitária Pan-Americana dirigida por Soper havia aprovado a mesma medida.) As ambiciosas metas de erradicação da malária haviam sido concebidas e promovidas no contexto de grande entusiasmo e otimismo diante da 
capacidade de o DDT ser amplamente aspergido para matar mosquitos e sobre o surgimento de novas drogas antimaláricas. Como argumentou Randall Packard, os Estados Unidos e seus aliados acreditavam que a erradicação global da malária conduziria o crescimento econômico e criaria mercados ultramarinos para a tecnologia e os bens manufaturados dos Estados Unidos (Packard, 1997; 1998). Constituiria apoio a governos locais, e aos norteamericanos que os apoiassem, e ajudaria a conquistar "corações e mentes" na batalha contra o comunismo. A campanha reproduziu as teorias de desenvolvimento da época através da promoção de tecnologias levadas de fora, sem realizar um maior esforço para atrair a participação da população local no seu planejamento ou na sua implementação. O modelo de assistência ao desenvolvimento ajustava-se perfeitamente aos esforços da Guerra Fria dos Estados Unidos em promover a modernização com reformas sociais limitadas administradas por um pequeno grupo de especialistas (Packard \& Brown, 1997).

Com o retorno da União Soviética e outros países comunistas em 1956, o equilíbrio político na Assembléia Mundial da Saúde mudou e Candau acomodou a alteração no poder. Durante os anos 60, a erradicação da malária estava passando por sérias dificuldades no campo; no final, viria a sofrer fracassos colossais e constrangedores. Em 1969, a Assembléia Mundial da Saúde realizada em Boston declarou não ser viável erradicar a malária em muitas partes do mundo e iniciou um lento processo de reversão, retornando novamente a uma agenda mais antiga de controle dessa doença. Mesmo assim, a Assembléia de 1969 enfatizou a necessidade de desenvolver sistemas rurais de saúde e de integrar o controle da malária a serviços gerais de saúde.

Quando a União Soviética retornou à OMS em 1956, seu representante na Assembléia, o então ministro da Saúde da URSS, argumentava ser cientificamente viável, socialmente desejável e economicamente vantajoso tentar erradicar mundialmente a varíola (Glynn \& Glynn, 2004). A URSS queria deixar sua marca na saúde internacional e Candau, reconhecendo a mudança no equilíbrio de poder, estava disposto a cooperar. A URSS concordou em oferecer $25 \mathrm{mi}-$ lhões de doses de vacina congelada a vácuo, e Cuba, 2 milhões de doses de vacina; em 1959, a Assembléia Mundial da Saúde comprometeu-se com um programa global de erradicação da varíola.

Na década de 1960, avanços tecnológicos - injetáveis modernos e agulhas bifurcadas - tornaram o processo de vacinação muito mais barato, fácil e eficaz. O interesse dos Estados Unidos na erradicação da varíola aumentou acentuadamente e, em 1965, Lyndon Johnson instruiu a delegação dos Estados Unidos na Assembléia Mundial da Saúde a prometer o apoio norte-americano a um programa internacional de erradicação da varíola em todo o 
planeta (ibidem, p. 198). Embora se houvesse registrado um importante progresso durante a década anterior, a doença mantinhase endêmica em mais de trinta países. Em 1967, agora com o apoio dos mais importantes atores mundiais, a OMS lançou o Programa Intensificado de Erradicação da Varíola. Esse programa, um esforço internacional liderado pelo norte-americano Donald A. Henderson, no qual participou ativamente o brasileiro Ciro de Quadros, seria, ao final, surpreendentemente bem-sucedido (Foege, 1998; Henderson, 1998; Henderson, Arita, Jezek \& Ladnyi, 1988).

\section{A promessa e os perigos da atenção primária à saúde, 1973-1993}

No interior da OMS, sempre houve tensões entre as abordagens social e econômica da saúde da população, de um lado, e a abordagem focada em tecnologia e doenças, de outro. Essas tendências não são necessariamente contraditórias, embora tenham estado freqüentemente em disputa. A ênfase em uma ou outra aumentou ou diminuiu ao longo do tempo, dependendo do equilíbrio de poder mais amplo, das mudanças de interesses dos atores internacionais, dos compromissos intelectuais e ideológicos de indivíduoschave, e do modo como todos esses fatores interagem com o processo de decisão de políticas de saúde.

Durantes as décadas de 1960 e 1970, as mudanças na OMS foram significativamente influenciadas por um contexto político marcado pela emergência de nações africanas descolonizadas, a disseminação de movimentos nacionalistas e socialistas, e novas teorias de desenvolvimento que enfatizavam o crescimento sócioeconômico integral de longo prazo preferentemente à intervenção tecnológica de curto prazo. Nos primeiros anos da OMS as nações africanas não haviam desempenhado um papel preponderante porque eram, em sua grande maioria, ainda colônias ou territórios dominados por países Europeus que assumiam sua representação nas Assembléias Gerais da Saúde. Reunidas em organizações tais como o Movimento dos Países Não-Alinhados, países em desenvolvimento criaram a Conferência das Nações Unidas para o Comércio e o Desenvolvimento - Unctad, onde argumentavam vigorosamente pela adoção de condições mais justas no comércio e por financiamentos mais generosos para o desenvolvimento (Bhagwati, 1977; Rothstein, 1979). Em Washington (DC), políticas mais liberais sucederam o conservadorismo dos anos 50, com o movimento dos direitos civis e outros movimentos sociais forçando mudanças nas prioridades nacionais.

Esse contexto político em transformação refletiu-se em mudanças correspondentes no interior da OMS. Nos anos 60, a OMS reconheceu que a criação e a melhoria na infra-estrutura de saúde, 
especialmente no campo, era pré-requisito para o sucesso dos programas de controle da malária, especialmente na África. Em 1968, Candau requisitou um plano abrangente e de abordagem integrada para serviços de atendimento preventivo e curativo. Um representante soviético requisitou um estudo organizacional de métodos de promoção do desenvolvimento de serviços básicos de saúde (Litsios, 2002). Em janeiro de 1971, a comissão da diretoria executiva concordou com a realização desse estudo, e seu resultado foi apresentado à Assembléia em 1973 (WHO, 1972; 1973). Recentemente Socrates Litsios tem examinado muitos dos passos da transformação da abordagem da OMS, de um modelo mais antigo de serviços de saúde, no que viria a ser a abordagem de "Atenção primária à saúde" (Litsios, 2002; 2004). Esse novo modelo utilizou-se do pensamento e das experiências de organizações não-governamentais ONGs - e de médicos missionários atuantes na África, Ásia e América Latina junto às populações locais. Também ganhou projeção com o retorno da China à ONU em 1973 e o interesse amplamente disseminado pelos "médicos de pés descalços" chineses, sobre os quais se relatava estarem transformando as condições de saúde das populações das áreas rurais. Essas experiências ressaltaram a urgência de uma perspectiva de "Atenção primária à saúde" que incluísse o treinamento de trabalhadores comunitários de saúde e a resolução de problemas econômicos e ambientais básicos (Bryant, 1969; Taylor, 1976; Newell, 1975; Cueto, 2004b; Litsios, 2004).

Essas novas tendências estavam agora incorporadas na OMS por Halfdan T. Mahler, um carismático dinamarquês que serviu como diretor geral de 1973 a 1988 e que havia combatido a tuberculose no Equador e na Índia. Sob pressão do delegado soviético na Diretoria Executiva, Mahler concordou em realizar uma grande conferência sobre a organização de serviços de saúde em AlmaAta, uma província remota na antiga União Soviética que em parte se assemelhava às condições sociais dos países em desenvolvimento. Mahler relutou, inicialmente, porque não concordava com a abordagem altamente centralizada e medicalizada da União Soviética com relação à oferta de serviços de saúde (Litsios, 2002). E, embora a União Soviética tenha alcançado seu intento com a realização do evento no seu território, a conferência resultante, ocorrida em setembro de 1978, refletiu mais de perto a visão de Mahler do que a dos soviéticos. A "Declaração de Atenção Primária à Saúde" e a meta "Saúde para todos no ano 2000" defendiam uma abordagem "intersetorial" e multidimensional para a saúde e o desenvolvimento sócio-econômico, enfatizavam o uso de "tecnologia apropriada", quer dizer, não custosa e adaptada ao meio social onde seria implementada, e instavam pela ativa participação comunitária no atendimento à saúde e na educação de saúde em todos os níveis (Declaration of Alma-Ata, 1978). 
O peruano David Tejada de Rivero, então funcionário da OMS, argumentou que "é lamentável que, em seguida, a impaciência de algumas agências internacionais, tanto da ONU quanto privadas, e sua ênfase no alcance tangível de resultados, ao invés de promover mudança ... tenha levado a importantes distorções do conceito original de atenção primária à saúde" (Tejada de Rivero, 2003). Alguns governos, agências e indivíduos entenderam a visão idealista da OMS como "irrealista" e inalcançável. O processo de redução do idealismo de Alma-Ata a um conjunto prático de intervenções técnicas mais facilmente implementáveis, geridas e mensuradas, teve início em 1979 numa pequena conferência com forte influência dos Estados Unidos, realizada em Bellagio, na Itália, e financiada pela Fundação Rockefeller, com apoio do Banco Mundial. Entre os participantes incluíam-se o presidente do Banco Mundial, o vice-presidente da Fundação Ford, o administrador da Usaid e o secretário-executivo da Unicef (Black, 1996; 1986). ${ }^{4}$ A reunião de Bellagio focalizou um conceito alternativo ao articulado em Alma-A ta - "Atenção primária seletiva à saúde" -, construído sobre a noção de intervenções técnicas pragmáticas e de baixo custo, que fossem limitadas em seu escopo e facilmente monitoradas e avaliadas. Graças especialmente à Unicef, que a partir de 1980 teve a liderança carismática de Jim Grant, a "Atenção primária seletiva à saúde" foi logo operacionalizada sob o acrônimo GOBI - Growth monitoring to fight malnutrition in children, Oral rehydration techniques to defeat diarrheal diseases, Breastfeeding to protect children; and Immunizations (Monitoramento do crescimento para combater a desnutrição em crianças, Técnicas de re-hidratação oral para combater doenças diarréicas, Amamentação para proteção das crianças, e Imunização) (Unicef, 1983; Cueto, 2004b).

Na década de 1980, a OMS teve de levar em consideração a crescente influência do Banco Mundial, instituição que aumentou seu interesse e protagonismo na saúde internacional durante o período em que Robert McNamara foi seu presidente (1968-1981). O Banco havia sido criado em 1946, inicialmente para apoiar a reconstrução da Europa, e posteriormente expandiu seu mandato para oferecer empréstimos, subvenções e assistência técnica a países em desenvolvimento. No início, financiou grandes investimentos em capital físico e infra-estrutura, mas nos anos 70 começou a investir em controle populacional, saúde e educação, com ênfase no controle populacional (Ruger, 2005). O Banco Mundial aprovou seu primeiro empréstimo para planejamento familiar em 1970. Em 1979, criou o Population, Health, and Nutrition Department (Departamento para População, Saúde e Nutrição) e adotou uma política de financiamento tanto para programas exclusivamente de saúde, quanto de componentes de saúde de outros projetos.

No seu World Development Report (Relatório sobre o desenvolvimento mundial) de 1980, o Banco argumentou que tanto a desnu- 
trição quanto a doença poderiam ser tratados mediante ação governamental direta - com a sua assistência (World Bank, 1980). Sugeriu também que a melhoria da saúde e da nutrição poderia acelerar o crescimento econômico, oferecendo assim um bom argumento para gastos no setor social. Na medida em que o Banco Mundial começou a fazer empréstimos diretos para serviços de saúde, requereu o uso mais eficaz dos recursos disponíveis e levantou a discussão sobre o papel dos setores público e privado no financiamento do atendimento à saúde. O Banco favorecia mercados livres e minimizava o papel dos governos nacionais (World Bank, 1987). No contexto do endividamento de muitos países em desenvolvimento e da crescente escassez de recursos para gastos com saúde, a adoção de medidas de "ajuste estrutural" pelo Banco Mundial, no exato momento da erupção da epidemia da Aids, provocou severas críticas, mas também ressaltou a nova influência do Banco.

Em contraste com a crescente autoridade do Banco Mundial, nos anos 80 o prestígio da Organização Mundial da Saúde estava começando a diminuir. Um sinal de dificuldade foi o voto, em 1982, da Assembléia Mundial da Saúde pelo congelamento do orçamento da OMS (Godlee, 1994a, b). A isso seguiu-se a decisão dos Estados Unidos, em 1985, de pagar apenas 20 por cento da sua contribuição fixada para todas as agências da ONU, e de suspender sua contribuição ao orçamento regular da OMS, em parte como protesto contra o Essential Drug Program (Programa de Medicamentos Essenciais) da OMS, ao qual se opuseram as principais empresas farmacêuticas baseadas nos Estados Unidos (ibidem, p. 1492). Esses eventos ocorreram em meio a crescentes tensões entre a OMS, a Unicef e outras agências, e à controvérsia sobre 'atenção primária à saúde' - 'seletiva' versus 'integral'. Como parte de um rancoroso debate público conduzido nas páginas de Social science and medicine, em 1988, Kenneth Newell, alto funcionário da OMS e arquiteto do Comprehensive Primary Health Care (Atenção primária integral à saúde), chamou o Selective Primary Health Care (Atenção primária seletiva à saúde) de "ameaça ... [que] pode ser entendida como uma contra-revolução" (Newell, 1988).

Em 1988, o mandato de quinze anos de Mahler como diretor geral da OMS terminou. Inesperadamente, Hiroshi Nakajima, pesquisador japonês que havia sido diretor da OMS no Escritório Regional do Pacífico Ocidental, em Manila, foi eleito novo diretor geral (Lewis, 1988).

\section{Crise na OMS, 1988-1998}

Primeiro cidadão japonês eleito para a direção de uma agência da ONU, Nakajima rapidamente tornou-se o mais controverso diretor geral na história da OMS. Sua nomeação não teve o apoio 
dos Estados Unidos nem de vários países europeus e latinoamericanos, e sua atuação pouco contribuiu para diminuir as dúvidas. Nakajima tentou lançar várias iniciativas importantes tabaco, vigilância global sobre doenças, parcerias público-privadas - mas fortes críticas persistiram e levantaram questionamentos sobre seu estilo autocrático e sobre sua gestão medíocre, sua inabilidade para comunicar-se eficientemente e, pior que tudo, favoritismo e acusações de corrupção.

Outro fator que minou o prestígio de Nakajima foi seu conflito com Jonathan Mann, um prestigiado jovem médico norte-americano que havia conseguido em pouco tempo recursos e apoios substanciais para o primeiro Programa Global da OMS contra a Aids. Desse modo, Mann estava contribuindo para fazer dessa doença um tema prioritário na agenda política mundial. Contudo, em um conflito pelo qual aparentemente Nakajima foi responsabilizado, Mann renunciou dramaticamente de seu posto na OMS, ao se sentir pressionado pela intenção do diretor geral de restringir suas funções e minimizar sua popularidade (Hilts, 1990). Pouco depois se formou a Onusida como agência na qual participava a OMS, mas associada com outros organismos. Mann seguiu lutando pelos direitos humanos e combatendo a Aids/HIV, e foi nomeado professor na Universidade Harvard.

Outro sintoma dos problemas da OMS no final dos anos 80 foi o crescimento de fundos extra-orçamentários. Como observou Gill Walt, da London School of Hygiene and Tropical Medicine (Escola de Higiene e Medicina Tropical de Londres), houve uma mudança crucial da dependência predominante do 'orçamento regular' da OMS - obtido da contribuição dos Estados membros, baseada em tamanho da população e PIB - para uma dependência grandemente aumentada de fundos 'extra-orçamentários' resultantes de doações de agências multilaterais ou países 'doadores' (Walt, 1993). Em 1986 e 1987, fundos extra-orçamentários de 437 milhões de dólares quase haviam atingido o montante do orçamento regular, de $543 \mathrm{mi}$ lhões de dólares. No início da década de 1990, fundos extraorçamentários haviam ultrapassado em 21 milhões de dólares o orçamento regular, contribuindo assim com 54 por cento do orçamento total da OMS. Imensos problemas surgiram para a Organização em virtude dessa mudança orçamentária. Prioridades e políticas eram pretensamente definidas pela Assembléia Mundial da Saúde, composta por todos os países membros, mas esta, então numericamente dominada por países pobres e em desenvolvimento, tinha autoridade somente sobre o orçamento regular, congelado desde o início dos anos 80. Países doadores ricos e agências multilaterais, como o Banco Mundial, podiam ter amplo controle sobre o uso dos fundos extra-orçamentários com os quais contribuíam. Assim, com efeito, estes últimos criaram vários programas 'verticais' mais 
ou menos independentes do restante dos programas da OMS e da estrutura de tomada de decisões. O dilema para a Organização era que, embora os fundos extra-orçamentários se somassem ao orçamento geral, "eles aumentam as dificuldades de coordenação e continuidade, causam imprevisibilidade financeira, e uma grande dependência da satisfação de doadores específicos" (ibidem, p. 129).

Fiona Godlee publicou uma série de artigos em 1994 e 1995 baseada na crítica de Walt (Godlee, 1994a, b; 1995). Sua conclusão é negativa: "a OMS está inserida num ciclo de declínio, com doadores expressando sua falta de confiança na gestão central ao alocarem recursos fora do controle da administração. Isso tem impedido a OMS [de desenvolver] ... respostas integradas às necessidades de longo prazo dos países".

No final dos anos 80 e início dos 90, o Banco Mundial deslocouse confiantemente para ocupar o vácuo criado por uma crescente ineficiência da OMS. Funcionários da OMS foram incapazes ou não tiveram determinação para responder à nova política econômica internacional estruturada em torno de abordagens neoliberais quanto a economia, comércio e política (Brown, 1997; “World Bank's cure for donor fatigue", 1993; Zwi, 2000). O Banco sustentava que os sistemas de saúde existentes freqüentemente causavam desperdício de recursos financeiros e humanos, eram ineficientes e ineficazes, e argumentava a favor de uma maior presença do setor privado na provisão de atenção à saúde, com a redução do envolvimento público no oferecimento de serviços de saúde (World Bank, 1987).

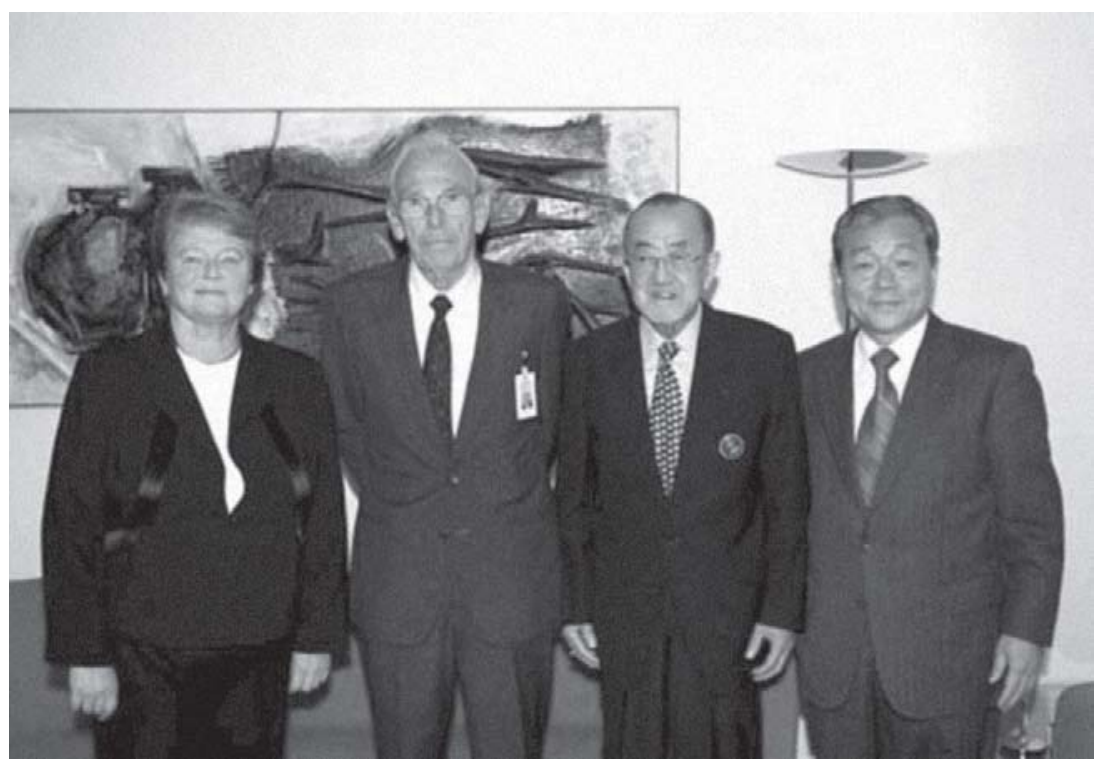

Dr. Lee Jong-wook, primeiro da direita para esquerda e três Diretores-Gerais Eméritos da OMS: Dr G.H.Brundtland, Dr. H. Mahler e Dr. H. Nakajima. Arquivo da Organização Mundial da Saúde, Genebra, Suíça. 
As políticas e práticas do Banco cercavam-se de controvérsias, mas não havia dúvida de que, no início dos anos 90, tornara-se uma força dominante na saúde internacional. A grande 'vantagem comparativa' do Banco recaía na sua capacidade de mobilizar grandes somas de recursos financeiros; em 1990, os empréstimos do Banco para saúde ultrapassaram o orçamento total da OMS, e, ao final de 1996, a carteira cumulativa de empréstimos do Banco em saúde, nutrição e população alcançara 13,5 bilhões de dólares. Mesmo assim, o Banco Mundial reconhecia que, embora tivesse grande influência e força econômica, a OMS ainda dispunha de considerável conhecimento técnico em matéria de saúde e medicina. Isso se refletiu claramente no relatório amplamente difundido World Development Report, 1993: investing in health (Relatório sobre o desenvolvimento mundial, 1993: investindo em saúde), documento que teve grande influência, no qual dava crédito à OMS, "parceiro integral do Banco Mundial em todos os estágios de preparação do Relatório" (World Bank, 1993, p. iii-iv). As circunstâncias indicavam ser vantajoso para ambas as partes que o Banco e a OMS trabalhassem em conjunto.

\section{A OMS adota a 'saúde global'}

Esse é o contexto no qual a OMS começou a remodelar-se na coordenação, no planejamento estratégico e na liderança de iniciativas de 'saúde global'. Em janeiro de 1992, os 31 membros da Diretoria Executiva da Assembléia Mundial da Saúde decidiram nomear um "grupo de trabalho" para recomendar de que maneira a OMS poderia ser mais eficaz no trabalho de saúde internacional à luz da "mudança global" que rapidamente tomava conta do mundo. A Diretoria Executiva poderia estar respondendo, em parte, à Children's Vaccine Initiative (Iniciativa de vacinação infantil), percebida no âmbito da OMS como uma tentativa de 'golpe' da Unicef, do Banco Mundial, do Programa de Desenvolvimento da ONU, da Fundação Rockefeller e de diversos outros atores, visando controlar o desenvolvimento de vacinas. ${ }^{5} \mathrm{O}$ relatório final do grupo de trabalho, de maio de 1993, recomendou que a OMS - se quisesse manter a liderança no setor de saúde - fizesse uma revisão na sua gestão fragmentária dos programas global, regional e por país, diminuir a competição entre programas do orçamento regular e extra-orçamentários e, sobretudo, aumentar a ênfase, no interior da OMS, nos temas de saúde global e no papel da OMS de coordenador nesse âmbito (Stenson \& Sterky, 1994), p. 242).

Até aquele momento, a expressão 'saúde global' havia sido usada esporadicamente e, fora da OMS, em geral por pessoas politicamente à esquerda, com várias agendas 'mundiais'. Em 1990, G. A. Gellert, do International Physicians for the Prevention of Nuclear War 
(Médicos internacionais para a prevenção da guerra nuclear), havia levantado a questão da análise da "interdependência da saúde global" (Gellert, 1990). No mesmo ano, Milton e Ruth Roemer argumentaram que maiores avanços na 'saúde global' dependeriam da expansão de serviços públicos de saúde, preferivelmente os privados (Roemer \& Roemer, 1990). Outra importante fonte para a expressão 'saúde global' foi o movimento ambientalista e, em especial, os debates sobre degradação ambiental mundial, aquecimento global, e seus efeitos potencialmente devastadores sobre a saúde humana. ${ }^{6}$ Em meados dos anos 90 havia considerável literatura sobre as ameaças para a saúde global. Nos Estados Unidos, teve início a publicação de um novo jornal do CDC - Centers for Disease Control and Prevention, ${ }^{7}$ Emerging infectious diseases (Doenças infecciosas emergentes), e o ex-diretor da CDC William Goege começou a referir-se a "ameaças de doenças infecciosas globais" (Morse, 1995). Em 1997, a Diretoria de Saúde Internacional do Institute of Medicine publicou um relatório intitulado America's vital interest in global health: protecting our people, enhancing our economy, and advancing our international interests (O interesse vital da América em saúde global: protegendo nosso povo, incrementando nossa economia e levando adiante nossos interesses internacionais). Em 1998, o CDC publicou Preventing emerging infectious diseases: a strategy for the $21^{\text {st }}$ century (Prevenindo doenças infecciosas emergentes: uma estratégia para o século XXI), seguida em 2001 pela publicação do Institute of Medicine, Perspectives on the Department of Defense global emerging

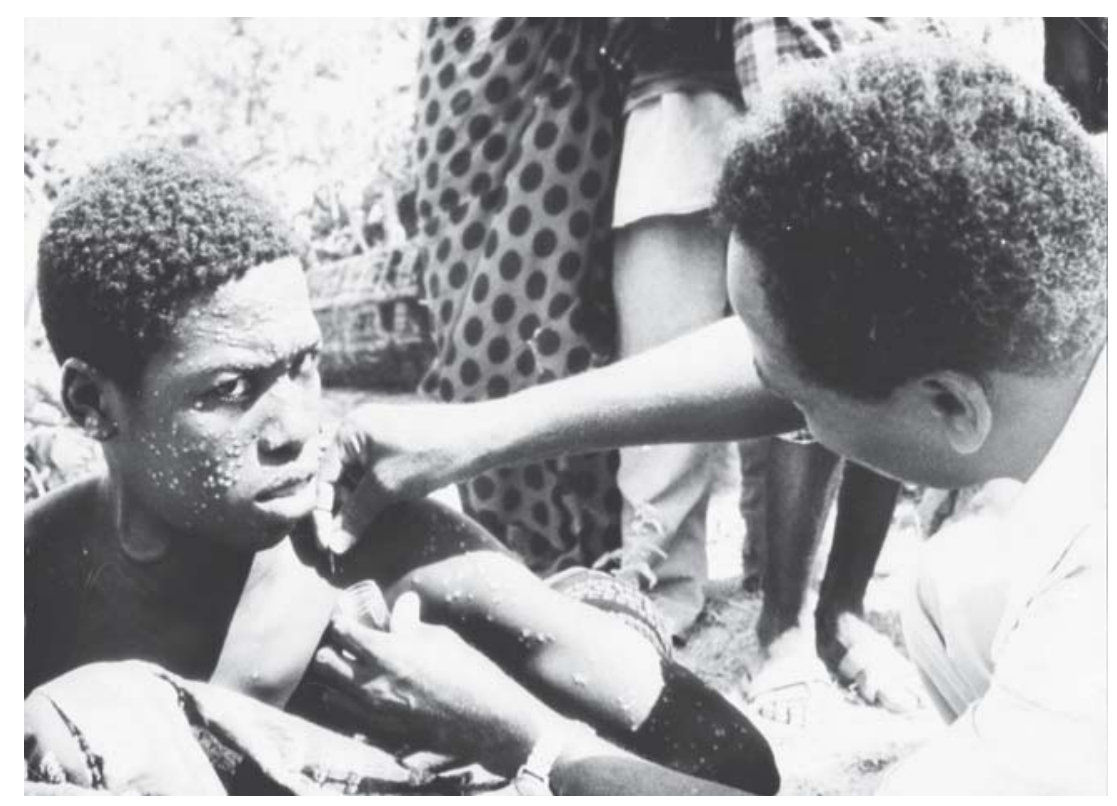

Profissional da OMS na campanha de erradicação da varíola na África, década de 1970. Foto de J. Rist, Biblioteca Nacional de Medicina, Bethesda, Estados Unidos. 
infections surveillance and response system (Perspectivas sobre o sistema de supervisão e resposta a infecções globais emergentes do Departamento de Defesa) (Krause, 1998; CDC, 1998; Brachman et al., 2001). Livros na lista dos mais vendidos e novas revistas estavam repletos de estórias sobre o vírus Ébola e o Vírus do Oeste do Nilo, tuberculose ressurgente e resistente aos medicamentos tradicionais, como também sobre a ameaça de bioterrorismo (por exemplo, Garrett, 1994). A mensagem era clara: havia uma ameaça palpável de doença global.

Em 1998, a Assembléia Mundial da Saúde buscou fora dos quadros da OMS uma liderança que pudesse restaurar a credibilidade da organização e trazer-lhe uma nova visão: Gro Harlem Brundtland, médica e profissional da saúde pública que havia sido primeiraministra da Noruega. Brundtland tinha imenso conhecimento para desempenhar a função. Na década de 1980, havia presidido a World Commission on Environment and Development (Comissão mundial para o meio ambiente e o desenvolvimento) da ONU, e produzira o Brundtland Report (Relatório Brundtland), que conduziu à Conferência Mundial de Meio Ambiente de 1992. Tinha familiaridade com o pensamento global do movimento ambientalista, e uma compreensão ampla e clara sobre a relação entre saúde, meio ambiente e desenvolvimento (Altman, 1992).

Brundtland estava determinada a posicionar a OMS como um importante ator no cenário global, a ir além de ministros da saúde e a ganhar assento à mesa quando decisões estivessem sendo tomadas (Kickbusch, 2000). Queria remodelar a OMS como uma organização que efetivamente cumprisse a missão para a qual havia sido criada, capaz de monitorar e influenciar outros atores no cenário global. Criou a Comissão sobre Macroeconomia e Saúde, presidida pelo economista Jeffrey Sachs, da Universidade Harvard, incluindo ex-ministros de finanças e funcionários do Banco Mundial, Fundo Monetário Internacional, Organização Mundial do Comércio e Programa das Nações Unidas para o Desenvolvimento, assim como lideranças da saúde pública. A Comissão publicou um relatório, em dezembro de 2001, que argumentava ser essencial a melhoria da saúde para o crescimento econômico dos países em desenvolvimento (WHO, 2001; ver também Waitzkin, 2003). O relatório identificou um conjunto de prioridades relativas a doenças que requeriam intervenção específica.

Brundtland também começou a fortalecer a situação financeira da OMS, especialmente pela organização de 'parcerias globais' e 'fundos globais', para reunir 'cotistas' - doadores privados, governos, e agências bilaterais e multilaterais - para concentrar-se em alvos específicos (por exemplo, Roll Back Malaria, em 1998, Global Alliance for Vaccines and Immunization, GAVI, em 1999, e Stop TB, em 2001). ${ }^{8}$ Eram programas semi-autônomos, aportando fundos 
externos substanciais, freqüentemente na forma de parcerias públicoprivadas (Reid \& Pearce, 2003). Um participante muito importante nessas 'PPPs' foi a Bill \& Melinda Gates Foundation, que aportou mais de 1,7 bilhão de dólares entre 1998 e 2000 para um programa internacional de prevenção ou eliminação de doenças nos países mais pobres do mundo através, sobretudo, de programas de vacinas e imunização (McCarthy, 2000). Em poucos anos, foram criadas cerca de setenta 'parcerias de saúde global'.

Os críticos das parcerias público-privadas argumentavam que as colaborações com o setor comercial poderiam subordinar os valores e a missão da OMS, modificar as prioridades organizacionais, recriar programas verticais desarticulados entre si em meio a sistemas de saúde fragmentados e afastar-se da abordagem compreensiva dos serviços de saúde, caminhando para um foco mais restrito em "doenças prioritárias" (Buse \& Waxman, 2001). Outra crítica à proliferação das parcerias público-privadas era que elas tendiam a fragmentar a estrutura financeira e de governança da saúde internacional, criando duplicação em determinadas áreas e nenhuma atenção em outras.

A gestão de Brundtland como diretora geral não foi isenta de defeitos nem livre de críticas. Algumas iniciativas creditadas à sua administração foram, de fato, iniciadas na gestão de Nakajima (por exemplo, Framework Convention on Tobacco Control - Convenção para referência sobre o controle do tabaco, da OMS), outras podem ser vistas hoje com algum ceticismo (Comissão sobre macroeconomia, Roll Back Malaria), e outras, ainda, não teriam recebido atenção suficiente de sua administração (atenção primaria à saúde, HIV/ Aids, saúde e direitos humanos, e saúde infantil). Entretanto, poucos contestariam a assertiva de que Brundtland alcançou seu principal objetivo, o de reposicionar a OMS como um ator digno de crédito e altamente visível no âmbito das rápidas mudanças da saúde global.

\section{Conclusão}

Podemos agora retornar, brevemente, às questões com as quais este ensaio foi iniciado: como pode uma perspectiva histórica nos ajudar a compreender a emergência da terminologia 'saúde global', e qual foi o papel da OMS como agente desse desenvolvimento? As respostas básicas derivam do fato de que a OMS, em vários momentos ao longo de sua história, alternadamente liderou, refletiu, e tentou acomodar-se a mudanças e desafios mais amplos no mundo em constante transformação da saúde internacional. Nos anos 50 e 60, passou de uma ênfase estreita na erradicação da malária e em campanhas verticais contra doenças, pelas quais se enfatizava o uso de novas tecnologias, para um interesse mais amplo no desen- 
volvimento dos serviços de saúde e uma concentração emergente na erradicação da varíola, quando mudanças na biologia, na economia e em grandes forças políticas transformaram as relações internacionais e a saúde pública. Nos anos 70 e 80, a OMS desenvolveu o conceito de 'atenção primária à saúde', mas depois mudou para uma promoção pragmática da 'atenção primária seletiva à saúde', na medida em que complexas mudanças alcançaram a dinâmica intra-organizacional e inter-organizacional e alteraram a ordem econômica e política internacional. Na década de 1990, a OMS tentou ocupar a liderança em uma preocupação emergente com 'saúde global' como uma estratégia organizacional que prometia sobrevivência, e mais, renovação. Porém, assim como não inventou as agendas de erradicação ou de atenção primária, a OMS também não inventou 'saúde global'. Outras forças, maiores, foram responsáveis por isso. De todo modo, a OMS certamente ajudou a promover o interesse em 'saúde global' e contribuiu significativamente para a disseminação de novos conceitos e de um novo vocabulário. Nesse processo, tinha esperança de conseguir, como sugeriram Yacht e Bettcher em 1998, a recuperação de um papel de coordenação e liderança. Se o reposicionamento organizacional da OMS servirá para restabelecer sua posição como administradora inquestionável da saúde da população mundial, e como essa missão será efetuada na prática, permanece, neste momento, uma questão em aberto.

A expressão 'saúde global' é, por vezes, apresentada como uma resposta puramente racional a eventos novos e ameaçadores na saúde pública, tais como epidemias internacionais atingindo tanto países ricos como países pobres, e a migração ilegal de populações. Como mostramos neste artigo, essa expressão emergiu como parte de um processo histórico e político mais amplo, em meio a um debate ainda não resolvido sobre a direção que deve tomar a saúde pública, no contexto de uma ordem mundial neoliberal, no qual a OMS viu seu papel, antes dominante, ser desafiado, e começou a reposicionar-se no âmbito de um conjunto de alianças de poder em transformação.

\section{NOTAS}

${ }^{1}$ Os autores agradecem a assistência de Julio Nunez na revisão e deste artigo. Este é uma versão ampliada do trabalho "The World Health Organization and the transition from international to global public health", American journal of public health, v. 96, n. 1, p. 62-72, 2006.

2 Uma pequena mostra de títulos recentes: Heymann \& Rodier, 1998; Woodward, Drager, Beaglehole \& Lipson, 2001; Walt, 1998; Kunitz, 2000; Lee, Buse \& Fustukian (2002).

3 Por exemplo, a Universidade Yale tem uma Division of Global Health (Divisão de Saúde Global) na School of Public Health (Escola de Saúde Pública); a Universidade Harvard tem um Center for Health and the Global Environment (Centro para a Saúde e o Meio Ambiente Global), e a London School of Hygiene and Tropical Medicine (Escola de Higiene e Medicina Tropical de Londres) tem o Center on Global Change and Health (Centro sobre Mudança e Saúde Global); o National Institute of Health (Instituto Nacional de Saúde, dos Estados Unidos) tem um plano 
estratégico sobre Emerging Infectious Diseases and Global Health (Doenças Infecciosas Emergentes e Saúde Global); no Simpósio do 35ํㅜ Aniversário do John E. Fogarty International Center, em maio de 2003, Gro Harlem Brundtland fez uma conferência sobre "Global Health: A Chalenge to Scientists" (Saúde global: um desafio para os cientistas); o Center of Disease Control and Prevention (Centro de Controle e Prevenção de Doenças) instituiu o Office of Global Health (Escritório de Saúde Global), e participou, em parceria com, entre outras instituições, a OMS, o Banco Mundial, a Unicef e a United States Agency for Internacional Development - Usaid (Agência Norte-Americana para o Desenvolvimento Internacional), da criação da Global Health Partnerships (Parcerias Saúde Global).

4 A Unicef foi criada em 1946 para dar assistência a crianças necessitadas na Europa, nas áreas devastadas pela guerra. Ao cessar a emergência, ampliou sua missão e concentrou recursos nas necessidades de crianças em países em desenvolvimento.

5 Para relato completo, ver Muraskin (1998).

6 Ver, por exemplo, Haines (1991); Haines, Epstein \& McMichael (1993); McMichael (1993); Last (1993); McMichael (1993); McMichael, Haines, Sloof \& Kovats (1996); McMichael \& Haines (1997).

7 Centers for Disease Control and Prevention (Centros para Controle e Prevenção de Doenças) - Department of Health and Human Services / U. S. Government.

8 RBM é um projeto cujo propósito é proporcionar melhoria das condições de saúde, concentrando-se no acesso e abastecimento dos medicamentos necessários para os pobres que sofrem de malária no mundo. Outras ações são a prevenção da transmissão com redes impregnadas de inseticidas e tratamento primário para buscar reduzir à metade a incidência dessa enfermidade no mundo. O presidente do Banco Mundial, o diretor executivo da Unicef e o administrador do UNDP (United Nations Development Program) fizeram um acordo, em outubro de 1998, sobre o início das atividades do RBM. A essas organizações se somaram novos participantes como os governos de países afetados pela malária, ONGs, representantes internacionais do setor de saúde e grupos de pesquisadores. A GAVI foi formada como uma aliança entre os setores privado e público, com a missão de cuidar da saúde e das vidas das crianças em particular e da população em geral mediante o uso intensivo de vacinas. Stop TB foi estabelecida com o propósito de eliminar a tuberculose como problema de saúde pública. Abarca uma rede de organizações internacionais, países, doadores dos setores público e privado, organizações governamentais e ONGs, bem como personalidades que têm expressado interesse de trabalhar visando alcançar essa meta.

\section{REFERÊNCIAS BIBLIOGRÁFICAS}

Altman, Lawrence K. 1992

Aykroyd, W. R. 1968

Balinska, Marta 1998

Banta, James E. 2001

Basch, Paul F. 1991

Bhagwati,

Jagdish N. (ed.) 1977

Birn, Anne-Emanuelle 1996

Black, Maggie 1996

Black, Maggie 1986
U. S. moves to replace Japanese head of WHO.

New York Times, Dec. 20, p. 1.

International health - a retrospective memoir. Perspectives in Biology and Medicine, p. 273-85.

For the good of humanity: Ludwik Rajchman, medical statesman. New York: Central European University Press.

From international to global health. Journal of community health, v. 26, p. 73-6.

A historical perspective on international health. Infectious Disease Clinics of North America, v. 5, p. 183-96.

The new international economic order: the north south debate. Cambridge (Mass): MIT Press.

Eradication, control or neither? Hookworm versus malaria strategies and Rockefeller public health in Mexico. Parassitologia, v. 40, p. 137-47.

Children first: the story of Unicef, past and present.

Oxford: Oxford University Press.

The children and the nations: the story of Unicef.

New York: Unicef. p. 114-40. 
Bourdreau, Frank G. 1944

Bourdreau, Frank G. 1929

Brachman, Philip S. et al. (ed.) 2001

Brown, $\mathrm{P}$ 1997

Bryant, John H. 1969

Bunyavanich, Supinda; Walkup, Ruth B. 2001

Buse, Kent; Waxman, Amalia 2001

Centers for Disease Control and Prevention (CDC)

1998

Commission on

Macroeconomics and

Health

2001

Cueto, Marcos

2004a

Cueto, Marcos

2004b

Cueto, Marcos (ed.) 1994

Declaration of Alma-Ata 1978

Deutsch, Alber 1958

Dubin, Martin David 1995

Farley, John 2003

Foege, William $\mathrm{H}$. 1998

Garrett, Laurie 1994

Gellert, G. A. 1990
International health work. In: Favis, Harriet Eager (ed.) Pioneers in world order: an American appraisal of the League of Nations.

New York: Columbia University Press. p. 193-207.

International Health.

American Journal of Public Health and the nation's health, v. 19, p. 863-78.

Perspectives on the Department of Defense Global Emerging Infections Surveillance and Response System.

Washington (DC): National Academy Press.

The WHO strikes mid-life crisis.

New scientist, v. 153, p. 12.

Health and the Developing World.

Ithaca (NY): Cornell University Press.

US public health leaders shift toward a new paradigm of global health. American journal of public health, v. 91, p. 1556-8.

Public-private health partnerships: a strategy for WHO.

Bulletin of the World Health Organization, v. 79, p. 748-54.

Preventing emerging infectious diseases: a strategy for the $21^{\text {st }}$ century.

Atlanta.

Macroeconomics and health: investing in health for economic development. Geneva: WHO

El valor de la salud: una historia de la Organización Panamericana de la Salud. Washington (DC): OPS.

The origins of primary health care and selective primary health care. American journal of public health, v. 94, p. 1864-74.

Missionaries of science: Latin America and the Rockefeller Foundation. Bloomington: Indiana University Press.

International Conference on Primary Health Care, Alma-Ata, USSR, 6-12 Sept. www.who.int/hpr/NPH/docs/declaration_almaata.pdf, acessado em 10 de abril de 2004.

The World Health Organization: its global battle against disease. New York: Public Affairs Committee.

The League of Nations Health Organization. In: Weindling, Paul (ed.) International health organizations and the movements, 1918-1939. Cambridge: Cambridge University Press. p. 56-80.

To cast out disease: A history of the International Health Division of the Rockefeller Foundation (1913-1951). Oxford: Oxford University Press.

Commentary: Smallpox eradication in West and Central Africa revisited. Bulletin of the World Health Organization, v. 76, p. 233-35.

The coming plague: newly emerging diseases in a world out of balance. New York: Farrar, Straus and Giroux.

Global health interdependence and the international physicians' movement. Journal of the American Medical Association, v. 264, p. 610-3. 
Glynn, lan;

Glynn, Jennifer 2004

Godlee, Fiona 1995

Godlee, Fiona $1994 a$

Godlee, Fiona 1994b

Haines, Andrew J. 1991

Haines, Andrew J.; Epstein, Paul R.; McMichael, Anthony J. 1993

Henderson, Donald A. 1998

Heymann, David L.;

Rodier, G. R. 1998

Henderson, Donald A.; Arita, Issao;

Jezek, Zdenëk;

Ladnyi, Ivan Dalinovich 1988

Hilts, Philip J. 1990

Howard-Jones, Norman 1978

Institute of Medicine 1997

Kickbusch, llona 2000

Krause,

Richard M. (ed.) 1998

Kunitz, Stephen J. 2000

Last, John M. 1993

Lee, Kelley; Buse, Ken; Fustukian, Suzanne (ed.) 2002

Lewis, Paul 1988

Litsios, Socrates 2004
The life and death of smallpox.

New York: Cambridge University Press. p. 194-6.

WHO's special programmes: undermining from above.

British medical journal, v. 310, p. 178-82.

WHO in crisis.

British medical journal, v. 309, p. 1424-8.

WHO in retreat: is it losing its influence?

British medical journal, v. 309, p. 1491-5.

Global warming and health.

British medical journal, v. 302, p. 669-70.

Global health watch: monitoring impacts of environmental change.

The Lancet, v. 342, Dec. 11, p. 1464-69.

Eradication: lessons from the past.

Bulletin of the World Health Organization, v. 76, Suppl 2, p. 17-21.

Global surveillance of communicable diseases.

Emerging infectious diseases, v. 4, p. 362-5.

Smallpox and its eradication.

Genebra: WHO

Leader in U.N.'s Battle on Aids reigns in dispute over strategy.

The New York Times, Mar 17, p. 1.

International public health between the two World Wars: the organizational problems. Geneva: WHO.

America's vital interest in global health: protecting our people, enhancing our economy, and advancing our international interests. Washington (DC): National Academy Press.

The development of international health priorities - accountability intact? Social science and medicine, v. 51, p. 979-89.

Emerging infections: biomedical research reports.

San Diego: Academic Press.

Globalization, states, and the health of indigenous peoples. American journal of public health, v. 90, p. 1531-9.

Global change: ozone depletion, greenhouse warming, and public health. Annual review of public health, v. 14, p. 115-36.

Health policy in a globalizing world.

Cambridge: Cambridge University Press.

Divided World Health Organization braces for leadership change. New York Times, May $1^{\text {st }}$, p. 20.

The Christian Medical Commission and the development of WHO's primary health care approach. American journal of public health, v. 94, p. $1884-93$. 
Litsios, Socrates 2002

McCarthy, Michael 2000

McMichael, Anthony. J. 1993

McMichael, Anthony J. 1993

McMichael, Anthony J.; Haines, Andrew 1997

McMichael, Anthony J.; Haines, Andrew J.;

Sloof, R.; Kovats, S. 1996

Morse, Stephen S. 1995

Muraskin, William 1998

Newell, Kenneth 1988

Newell, Kenneth W. 1975

Office International D'Hygiène Publique 1933

Organización Panamericana de la Salud - OPS 1992

Packard, Randall M. 1998

Packard, Randall M. 1997

Packard, Randall M.;

Brown, Peter J. 1997

Parran, Thomas 1958

Reid, Michael A.

Pearce, E. Jim 2003

Roemer, Milton;

Roemer, Ruth

1990

Rothstein, Robert L. 1979
The long and difficult road to Alma-Ata: a personal reflection. International journal of health services, v. 32, p. 709-32.

A conversation with the leaders of the Gates Foundation's Global Health Program: Gordon Perkin and William Foege. Lancet, v. 356, 8 July, p. 153-5

Planetary overload, global environmental change and the health of the human species. Cambridge: Cambridge University Press.

Global environmental change and human population health: a conceptual and scientific challenge for epidemiology.

International journal of epidemiology, v. 22, p. 1-8.

Global climate change: the potential effects on health.

British medical journal, v. 315, p. 805-9.

Climate change and human health.

Geneva: WHO.

Factors in the emergence of infectious diseases.

Emerging infectious diseases, v. 1, p. 7-15.

The politics of international health: the children's vaccine initiative and the struggle to develop vaccines for the Third World.

Albany (NY): State University of New York Press.

Selective Primary Health Care: the counter revolution.

Social science and medicine, v. 26, p. 903-6.

Health by the people.

Genebra: WHO.

Vingt-cinq ans d'activité de l'Office International D'Hygiène Publique, 1909-1933. Paris: Office International D'Hygiène Publique.

Pro Salute, Novi Mundi: historia de la Organización Panamericana de la Salud. Washington (DC): OPS.

'No other logical choice': global malaria eradication and the politics of international health in the post-war era. Parassitologia, n. 40, p. 217-29.

Malaria dreams: postwar visions of health and development in the Third World. Medical anthropology, v. 17, p.279-96.

Rethinking health, development and malaria: historicizing a cultural model in international health. Medical anthropology, v. 17, p. 181-94.

The first 12 years of WHO.

Public health reports, v. 73, p. 879-83.

Whither the World Health Organization?

The medical journal of Australia, v. 178, p. 9-12.

Global health, national development, and the role of government. American journal of public health, v. 80, p. 1188-92.

Global bargaining: UNCTAD and the Quest for a New International Economic Order. Princeton (NJ): Princeton University Press. 
Ruger, Jennifer Prah 2005

Seventh Meeting 1949

Siddiqi, Javed 1995

Silver, George 1998

Soper, Fred L. 1977

Stenson, Bo;

Sterky, Göran

1994

Taylor, Carl E. (ed.) 1976

Tejada de Rivero,

David A.

2003

Unicef

1983

U. S. House of Representatives 1971

Waitzkin, Howard 2003

Walt, Gill 1998

Walt, Gill 1993

Wilson, T. W. 1974

Woodward, David; Drager, Nick; Beaglehole, Robert; Lipson, Debra 2001

World Bank 1993

World Bank 1987

World Bank 1980 1993
Changing role of the world bank in global health in historical perspective. American journal of public health, v. 95, p. 60-70.

Seventh Meeting of the Executive Committee of the Pan American Sanitary Organization. Washington (DC), May 23-30, 1949.

Folder "Pan American Sanitary Bureau," R.G. 90-41, Box 9. Series Graduate School of Public Health, University of Pittsburgh Archives.

World health and world politics: the World Health Organization and the UN System. London: Hurst and Co.

International health services need an interorganizational policy.

American journal of public health, v. 88, p. 727-9; p.728.

Ventures. In: Duffy, John (ed.) World Health: the memoirs of Fred Lowe Soper. Washington (DC): PAHO.

What future WHO?

Health policy, v. 28 p. 235-56.

Doctors for the villages: study of rural internships in seven indian medical colleges. New York: Asia Publishing House.

Alma-Ata Revisited. Perspectives in health magazine: the magazine of the Pan American Health Organization, v. 8, p. 1-6.

The state of the world's children: 1982/1983.

New York: Oxford University Press.

The politics of global health, prepared for the Subcommittee on National Security Policy and Scientific Developments of the Committee on Foreign Affairs, U. S. House of Representatives. Washington (DC): U. S. Government Printing Office.

Report of the WHO Commission on Macroeconomics and Health: a summary and critique. The Lancet, v. 361, Febr. 8, p. 523-6.

Globalization of international health.

The Lancet, n. 351, Febr. 7, p. 434-7.

WHO under stress: implications for health policy.

Health policy, v. 24, p. 125-44.

World population and a global emergency. Washington (DC): Aspen Institute for Humanistic Studies, Program in Environment and Quality of Life.

Globalization and health: a framework for analysis and action. Bulletin of the World Health Organization, v. 79, p. 875-81.

World development report, 1993: investing in health.

Washington (DC).

Financing health services in developing countries: an agenda for reform. Washington (DC).

World Bank development report 1980.

Washington (DC).

"World Bank's cure for donor fatigue"

The Lancet, v. 342, July 10, p. 63-4. 
World Health

Organization - WHO s.d.

World Health Organization - WHO 1983

World Health

Organization - WHO 1973

World Health

Organization - WHO 1972

Yacht, Derek;

Bettcher, Douglas 1998a

Yacht, Derek;

Bettcher, Douglas 1998 b

Zubok, Vladislav; Pleshakov, Constantine 1996

Zwi, Anthony 2000
Information. Former Directors-General of the World Health Organization. Dr. Marcolino Gomes Candau. Disponível em www.who.int/archives/ who50/en/directors.htm, acessado em 24.07.2004.

In memory of Dr. M. G. Candau.

WHO Chronicle, v. 37, p. 144-7.

Executive Board, 49 $9^{\text {a }}$ Sessão, Genebra: WHO, documento EB49/SR/14

Rev., p. 218.

Organizational study of the Executive Board on methods of promoting the development of basic health services. Genebra: WHO, documento EB49/WP/6, p. 19-20.

The globalization of public health, I: Threats and opportunities.

American journal of public health, v. 88, p. 735-8.

The globalization of public health, II: The convergence of self-interest and altruism. American journal of public health, v. 88, p. 738-41.

Inside the Kremlin's Cold War, from Stalin to Khrushchev.

Cambridge: Harvard University Press.

Introduction to policy forum: the World Bank and international health. Social science and medicine, v. 50, p. 176. 